\title{
DnaK from Bifidobacterium animalis subsp. lactis is a surface-exposed human plasminogen receptor upregulated in response to bile salts
}

\author{
Correspondence \\ Patrizia Brigidi \\ patrizia.brigidi@unibo.it
}

Received 19 January 2010

Revised 12 February 2010

Accepted 17 February 2010

\author{
Marco Candela, ${ }^{1}$ Manuela Centanni, ${ }^{1}$ Jessica Fiori, ${ }^{1}$ Elena Biagi, ${ }^{1}$ \\ Silvia Turroni, ${ }^{1}$ Catia Orrico, ${ }^{2}$ Simone Bergmann, ${ }^{3}$ Sven Hammerschmidt ${ }^{4}$ \\ and Patrizia Brigidi ${ }^{1}$
}

\author{
${ }^{1}$ Department of Pharmaceutical Sciences, University of Bologna, Italy \\ ${ }^{2}$ Department of Pathological Anatomy Martinelli, S. Orsola-Malpighi, University of Bologna, Italy \\ ${ }^{3}$ Department of Microbial Pathogenicity, Helmholtz Centre for Infection Research GmbH, \\ Braunschweig, Germany \\ ${ }^{4}$ Department of Genetics of Microorganisms, Institute for Genetics and Functional Genomics, Ernst \\ Moritz Arndt University of Greifswald, Greifswald, Germany
}

\begin{abstract}
Bifidobacterium animalis subsp. lactis lives in the gastrointestinal tract of most mammals, including humans. Recently, for the probiotic strain B. animalis subsp. lactis BI07, a dosedependent plasminogen-binding activity was demonstrated and five putative plasminogen-binding proteins were identified. Here we investigated the role of surface DnaK as a $B$. animalis subsp. lactis BI07 plasminogen receptor. DnaK was visualized on the bacterial cell surface by transmission electron microscopy. The His-tagged recombinant DnaK protein showed a high affinity for human plasminogen, with an equilibrium dissociation constant in the nanomolar range. The capability to tolerate physiological concentrations of bile salts is a crucial feature for an intestinal symbiont micro-organism. By proteome analysis we demonstrated that the long-term exposure of $B$. animalis subsp. lactis $\mathrm{BI07}$ to bile salts results in the upregulation of important surface plasminogen receptors such as DnaK and enolase. Moreover, adaptation of $B$. animalis subsp. lactis BI07 to physiological concentrations of bile salts significantly increased its capacity to interact with the host plasminogen system. By enhancing the bacterial capacity to interact with the host plasminogen, the gut bile environment may facilitate the colonization of the human host by B. animalis subsp. lactis BI07.
\end{abstract}

\section{INTRODUCTION}

Human beings are in coevolution with a complex microbial community living in their gastrointestinal tract (GIT), the human intestinal microbiota (Gill et al., 2006; Ley et al., 2008). Active and metabolizing, the intestinal microbiota affects several host physiological features and has recently been considered as an integral component of the human physiology (Neish, 2009; Round \& Mazmanian, 2009). Bifidobacteria are obligate anaerobes in the Actinomycetales branch of the high-G $+\mathrm{C}$ Gram-positive bacteria and can be found in the human GIT as both autochthonous and allochtonous residents (Klijn et al., 2005). As inhabitants of the human gut they are only a minor component of the adult intestinal microbiota, but they largely predominate in

Abbreviations: Anti-Eno, anti-pneumococcal enolase antiserum; $\mathrm{BSH}$, bile salt hydrolase; EACA, $\varepsilon$-aminocaproic acid; GIT, gastrointestinal tract; PA, plasminogen activator(s); Plg, plasminogen; uPA, urokinase plasminogen activator. the gastrointestinal ecosystem of breast-fed infants until weaning (Ventura et al., 2009). The presence of bifidobacteria in the human GIT has been correlated with several health-promoting effects (Guarner \& Malagelada, 2003); however, there is only fragmentary information about their ecology, physiology and interaction with the host. Originally isolated from fermented milk (Meile et al., 1997), the species Bifidobacterium animalis subsp. lactis is commonly found in the guts of healthy adults and infants (Turroni et al., 2009). The complete genome sequence of three $B$. animalis subsp. lactis strains has been recently determined, and important insights into its genetic basis for the interaction with the host and the adaptation to the human gut environment have been provided (Barrangou et al., 2009). In particular, four genes with a possible role in the interaction with human epithelial cells have been identified, including two putative collagen-adhesion proteins, an elastin-binding protein and a fibronectin-binding protein. Another aspect of the B. animalis subsp. lactis- 
host interaction was revealed by the dose-dependent human plasminogen (Plg)-binding activity demonstrated for the BI07 and DSM 10140 strains (Candela et al., 2007).

$\mathrm{Plg}$ is the zymogen of plasmin, a trypsin-like serine protease with a broad substrate specificity. Plg is a singlechain glycoprotein with a molecular mass of $92 \mathrm{kDa}$ and comprises an $\mathrm{N}$-terminal pre-activation peptide $(\sim 8 \mathrm{kDa})$, five consecutive lysine-binding disulfide-bonded tripleloop kringle domains (K1-5), and a serine-protease domain containing the catalytic triad (Vassalli et al., 1991). It is produced mainly by hepatocytes; however, other tissue sources for Plg synthesis have been identified, including the intestine (Zhang et al., 2002). The active form, plasmin, is involved in fibrinolysis (Collen \& Verstraete, 1975), homeostasis and degradation of the extracellular matrix and basement membrane (Saksela \& Rifkin, 1988).

The capability to intervene with the Plg/plasmin system is a strategy for host colonization shared by several pathogens and commensals of the human GIT (Parkkinen \& Korhonen, 1989; Schaumburg et al., 2004; Lähteenmäki et al., 2005; Sijbrandi et al., 2005; Bergmann \& Hammerschmidt, 2007; Hurmalainen et al., 2007; Candela et al., 2008b). The binding of Plg to the bacterial cell surface depends on the presence of surface Plg-binding proteins which can interact with Plg by means of a C-terminal lysine residue(s) or/and lysine-enriched internal Plg-binding motive(s) (Bergmann et al., 2003, 2005; Bergmann \& Hammerschmidt, 2007; Candela et al., 2009; Sha et al., 2009). With the recruitment of human Plg on the cell surface, and its subsequent conversion to plasmin, the micro-organism acquires a surface-associated and host-derived proteolytic activity useful for facilitating migration across physical and molecular barriers and for responding to the nutritional demands during the colonization process (Lähteenmäki et al., 2005).

Similarly to the bifidobacterial species of human origin Bifidobacterium bifidum and Bifidobacterium longum, the probiotic species $B$. animalis subsp. lactis showed a dosedependent binding activity to human Plg (Candela et al., 2007). In the presence of host-derived Plg activators (PA), $\mathrm{Plg}$ captured on the B. animalis subsp. lactis cell surface was converted to plasmin, endowing the bacteria with a Plgdependent surface-associated plasmin activity effective in the degradation of physiological host substrates (Candela et al., 2008b). The complete inhibition of Plg binding to $B$. animalis subsp. lactis BI07 in the presence of the lysine analogue $\varepsilon$-aminocaproic acid (EACA) suggested that $\mathrm{Plg}$ binding is strongly dependent on lysine residues of surface $\mathrm{Plg}$ receptors. Five putative human Plg receptors were identified in the cell-wall fraction of $B$. animalis subsp. lactis strain BI07: DnaK, glutamine synthetase, enolase, bile salt hydrolase (BSH) and phosphoglycerate mutase (Candela et al., 2007). Analogously to other Gram-positive bacteria (Lähteenmäki et al., 2001), the B. animalis subsp. lactis Plg-binding proteins are highly conserved cytoplas- mic proteins that, expressed on the bacterial cell wall, acquire a secondary 'moonlighting' function different from the one exerted in the cytoplasm. The glycolytic enzyme enolase of B. animalis subsp. lactis BI07 was characterized with respect to surface localization and Plg-binding activity. Proving its role as a bifidobacterial Plg receptor, B. animalis subsp. lactis BI07 enolase showed a high affinity for human $\mathrm{Plg}$, with an equilibrium dissociation constant in the nanomolar range (Candela et al., 2009).

Adaptation and tolerance to physiological concentrations of bile salts (usually below $5 \mathrm{mM}$ ) is a vital factor for bacterial colonization of the human gut ecosystem (Ridlon et al., 2006; Sanchez et al., 2007). Bile salts are detergentlike compounds that are secreted into the intestine during digestion. Besides their primary role in the emulsification and absorption of fats, bile salts also exert a strong antimicrobial activity by inducing membrane damage and causing oxidative stress to DNA (Bernstein et al., 1999). The adaptation of $B$. animalis subsp. lactis to bile salts involves a global change in its protein expression profile (Sanchez et al., 2007), and the issue of whether these changes could affect the manner of interaction with the host has been addressed. Very recently, with the attempt to specifically investigate if bile adaptation could influence the Bifidobacterium-host interaction, Ruiz et al. (2009) studied the effect of bile salts on the B. longum cell-wall proteome. The authors reported that the surface Plg receptors enolase and glutamine synthetase were upregulated in response to bile salts, suggesting that the adaptation of B. longum to the bile environment triggers the production of bacterial factors for intestinal colonization.

Here we assessed the role of DnaK as a surface Plg receptor in B. animalis subsp. lactis BI07 and also analysed the effect of the adaptation to the bile salts environment on the Plgbinding capacity of this bacterium. Demonstrating the influence of bile adaptation on the $B$. animalis subsp. lactis-host interaction process, the bile salts treatment induced the upregulation of surface Plg receptors and significantly increased the ability of the bacteria to interact with the host Plg system.

\section{METHODS}

Bacterial strains, media and growth conditions. B. animalis subsp. lactis BI07 was cultured in MRS medium (Difco) supplemented with $0.05 \%(\mathrm{w} / \mathrm{v}) \mathrm{L}$-cysteine at $37{ }^{\circ} \mathrm{C}$ in anaerobic conditions, obtained by using Anaerocult A (Merck) in a jar. The bifidobacterial cells were grown for $18 \mathrm{~h}$ until they reached the stationary phase. Liquid MRS cultures were also performed with bile salts (oxgall; Difco) at the final concentrations of 1.2 and $3 \mathrm{~g}^{-1}$. Since human bile salts are difficult to retrieve, oxgall was used in this study. Oxgall has higher concentrations of bile salts and phospholipids and lower phospholipid/bile salts molar ratios than human bile (Coleman et al., 1979), whereas the ratio of glycine- to taurine-conjugated bile acids is similar (Hafkenscheid \& Hectors, 1975). Escherichia coli OneShot TOP10, BL21Star(DE3) and XL10-Gold strains were cultured at $37{ }^{\circ} \mathrm{C}$ in Luria-Bertani (LB) medium with shaking. MagicMedia (Invitrogen) was used for recombinant protein expression. 
Kanamycin $\left(50 \mu \mathrm{g} \mathrm{ml}^{-1}\right)$ was added as selective agent when appropriate.

Extraction of bifidobacterial cytoplasmic and cell-wall fractions. Cytoplasmic proteins were extracted as reported by Candela et al. (2009). Briefly, stationary-phase cells of B. animalis subsp. lactis BI07 were collected, washed in $50 \mathrm{mM}$ Tris/ $\mathrm{HCl}$ ( $\mathrm{pH} 7.6$ ), resuspended in $500 \mu \mathrm{l}$ TE buffer [ $50 \mathrm{mM}$ Tris/HCl $(\mathrm{pH} 7.6), 5 \mathrm{mM}$ EDTA], and $50 \mu \mathrm{l}$ Complete Protease Inhibitors Solution (Roche) was added. The suspension was sonicated and centrifuged for $10 \mathrm{~min}$ at 14000 r.p.m. $(22000 \mathrm{~g})$ at $4{ }^{\circ} \mathrm{C}$. The supernatant was collected and centrifuged for $2 \mathrm{~h}$ at 45000 r.p.m. $(176000 \mathrm{~g})$ at $4{ }^{\circ} \mathrm{C}$ by using a Beckman Ultracentrifuge L7-55. The supernatant, containing cytoplasmic proteins, was stored at $-20{ }^{\circ} \mathrm{C}$. Cell-wall proteins were extracted as reported by Hardie \& Williams (1998). Briefly, after

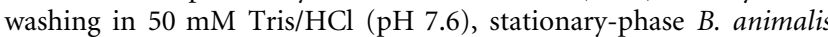
subsp. lactis BI07 cells were resuspended in $2 \mathrm{ml}$ protoplast buffer [50 mM Tris/ $\mathrm{HCl}$ ( $\mathrm{pH}$ 7.6), $1 \mathrm{M}$ sucrose, $1.4 \mathrm{mM}$ phenylmethylsulfonyl fluoride, $15 \mathrm{mg}$ lysozyme $\mathrm{ml}^{-1}$ ]. The suspension was incubated for $90 \mathrm{~min}$ at $37^{\circ} \mathrm{C}$ and centrifuged for $3 \mathrm{~min}$ at 4000 r.p.m. (1667 $\mathrm{g}$ ) at $4{ }^{\circ} \mathrm{C}$. The supernatant, containing the cell-wall proteins, was collected and stored at $-20{ }^{\circ} \mathrm{C}$.

Electron microscopy and immunoblot analysis. In order to visualize bifidobacterial DnaK on the B. animalis subsp. lactis BI07 cell surface, we performed pre-embedding immunogold experiments using whole bacterial cells. PBS-washed stationary-phase cells were adjusted to a concentration of $1 \times 10^{9}$ c.f.u. $\mathrm{ml}^{-1}$, resuspended in $100 \mu \mathrm{l}$ rabbit polyclonal anti-meningococcal DnaK antiserum (Knaust et al., 2007) diluted 1:100 in PBS-1\% BSAe (BSA), and incubated for $1 \mathrm{~h}$ at $25{ }^{\circ} \mathrm{C}$ under constant agitation. After two washings with $1 \mathrm{ml}$ PBS-1\% BSA, bacteria were resuspended in $25 \mu \mathrm{l}$ anti-rabbit IgG coupled to $10 \mathrm{~nm}$ gold particles (Auro Probe, GE Healthcare) diluted 1:5 in PBS-1\% BSA and incubated for $30 \mathrm{~min}$ at $25{ }^{\circ} \mathrm{C}$ with constant agitation. Bacteria were then collected and washed twice in PBS- $1 \%$ BSA and fixed with $2.5 \%$ glutaraldehyde in $0.1 \mathrm{M}$ cacodylate buffer for $4 \mathrm{~h}$ at $4{ }^{\circ} \mathrm{C}$. Transmission electron microscopy (TEM) processing was carried out as reported by Candela et al. (2007). Electron microscopic experiments were repeated four times. For immunoblot analysis, B. animalis subsp. lactis BI07 cytoplasmic or cell-wall proteins $(10 \mu \mathrm{g})$ were subjected to SDSPAGE with $12 \%$ polyacrylamide and blotted onto a nitrocellulose membrane (Bio-Rad) by using the Minitrans-Blot Electrophoretic Cell (Bio-Rad). Post-transfer, the membrane was blocked in a solution of $4 \%$ skim milk (Biolife) in TBS-T $(0.15 \%$ Tween 20 in TBS) and then incubated with anti-meningococcal DnaK antiserum (Knaust et al., 2007). Subsequently, the membrane was washed in TBS-T and incubated with the peroxidase-conjugated anti-rabbit IgG (GE Healthcare). After TBS-T washing, the membrane was incubated with ECL Plus (GE Healthcare), and the signal was detected by using the PhosphorImager Storm system (GE Healthcare).

Construction of a B. animalis subsp. lactis BI07 genomic library and screening for the dnaK gene. Three hundred micrograms of $B$. animalis subsp. lactis BI07 chromosomal DNA was partially digested with the restriction enzyme MboI. DNA fragments of about $20 \mathrm{~kb}$ were isolated by ultracentrifugation at 26000 r.p.m. for $24 \mathrm{~h}$ at $18{ }^{\circ} \mathrm{C}$ in a sucrose gradient (10-40\%), purified by precipitation with ethanol and ligated to BamHI-digested Lambda EMBL3 vector (Stratagene). Recombinant lambda phages were packaged by using the Lambda EMBL3/BamHI Gigapack III Gold Cloning kit (Stratagene). The library was amplified and titrated as described in the kit manual. A digoxigenin-labelled DNA probe targeting the $d n a K$ gene was obtained by amplifying the chromosomal DNA of B. longum NCC 2705 with the primer set L-DnaK (5'TTGGCACGTGCAGTTG-3') and R-DnaKwt (5'-TCACTTGTTGTCCTTGTCG- $3^{\prime}$ ) (designed based on the genome sequence of
B. longum NCC 2705) by using the DIG DNA Labelling and Detection kit (Roche). For screening, the phage library was plated on $120 \mathrm{~mm}$ NZY agar plates using E. coli XL1-Blue MRA P2 as host strain. Plates were blotted onto nylon membrane (Hybond-N +, GE Healthcare). Neutralization, denaturation, cross-linking, membrane hybridization with the DIG-labelled DNA probe, and detection were carried out as described by the DIG-DNA Labelling and Detection kit manual (Roche). Plaques corresponding to the positive signals in the original plates were cut out and resuspended in SM buffer to isolate the recombinant phage population. Phage DNA was extracted by using the Qiagen Lambda mini kit (Qiagen). Positive clones were sequenced and the sequence of the B. animalis subsp. lactis BI07 dnaK gene obtained.

Cloning, expression, purification of recombinant $B$. animalis subsp. lactis BI07 His $_{\mathbf{6}}$-DnaK. B. animalis subsp. lactis $\mathrm{BI} 07$ chromosomal DNA was used as template in a PCR for the amplification of the dnaK gene using the primer set DnaK-TOPO-L (5'-CACCATGGGACGCGCAGTTGGT- $\left.3^{\prime}\right)$ and DnaK-TOPO-R (5'TTACTTGTTGTCCTTGTCGTCGTC-3'). The PCR product was cloned in the expression vector pET200/D-TOPO (Invitrogen) to obtain the construct pDKwt. Cloning and expression of B. animalis subsp. lactis BI07 dnaK gene were carried out in E. coli TOP-10 and E. coli BL21Star(DE3) (Invitrogen), respectively. The His-tagged fusion B. animalis subsp. lactis BI07 DnaK was purified by affinity chromatography under native conditions on Ni-nitrilotriacetic acid resin according to the manufacturer's protocol (Invitrogen). The purified protein was dialysed at $4{ }^{\circ} \mathrm{C}$ using Spectra/Por membranes 6000-8000 Da (Spectrum Laboratories) and $20 \mathrm{mM}$ Tris, $120 \mathrm{mM}$ $\mathrm{NaCl}$ as dialysis buffer. The expression of the His-tagged recombinant protein $\mathrm{His}_{6}$-DnaK was verified by Western blot analysis with polyclonal anti-meningococcal DnaK antiserum (Knaust et al., 2007) and anti-HisTag antibody (Sigma).

Plg-binding analysis by solid-phase binding assay. Human Plg (Sigma-Aldrich) was biotinylated by using the EZ-Link Micro SulfoNHS-SS Biotinylation kit (Pierce). The solid-phase Plg-binding assay was performed as previously described by Sanderson-Smith et al. (2006, 2007). Microtitre plates (96-well; OptiPlate-96, Perkin Elmer) were coated with $150 \mathrm{nM}$ recombinant DnaK $(50 \mu \mathrm{l}$ in $0.1 \mathrm{M}$ $\mathrm{NaHCO}_{3}$ ) and incubated at $4{ }^{\circ} \mathrm{C}$. After washing with PBS, plates were blocked with $200 \mu \mathrm{l}$ BSA $2 \%$ in PBS for $1 \mathrm{~h}$ at $37^{\circ} \mathrm{C}$. After three PBS washings, increasing concentrations of biotinylated Plg $(2,6,18$, $36,55,110,166,250 \mathrm{nM}$ ) in PBS were added to the plates, in the presence or absence of 50 -fold molar excess of unlabelled Plg. Plg was allowed to bind to immobilized proteins for $2 \mathrm{~h}$ at $25^{\circ} \mathrm{C}$. Plates were washed three times in PBS and $50 \mu$ ExtrAvidin HRP conjugated (Sigma-Aldrich) diluted 1:5000 in PBS-1\% BSA was added to each well. Plates were incubated for $2 \mathrm{~h}$ at $25^{\circ} \mathrm{C}$ and washed four times with PBS and $0.05 \%$ Tween 20. One hundred microlitres of Chemiluminescent Peroxidase Substrate for ELISA (Sigma-Aldrich) was added to each well and luminescence was read by using the Victor $^{3} \mathrm{~V} 1420$ Multilabel Counter scanner (Perkin Elmer) and the software Wallac 1420 WorkStation. As negative control for Plg binding, BSA-coated wells were utilized. Plg-binding experiments were repeated in three biological replicates where, for each Plg concentration, three technical replicates were performed. To assess the involvement of lysine residues in the DnaK-Plg interaction, experiments were repeated in the presence of the lysine analogue $\varepsilon$-aminocaproic acid (EACA) $(0.5 \mathrm{M})$. Data were normalized against the highest and lowest luminescence value, and non-linear regression analysis was carried out by using GraphPad Prism (version 5.0). For calculation of the equilibrium dissociation constant $\left(K_{\mathrm{D}}\right)$, one- and two-site binding analyses were performed and the best-fit curve was chosen.

Two-dimensional gel electrophoresis and comparative analysis of protein expression. Experiments were carried out as 
reported by Candela et al. (2007). Briefly, $40 \mu \mathrm{g}$ samples of $B$. animalis subsp. lactis BI07 cell wall proteins were resolved by two-dimensional electrophoresis. Isoelectric focusing was carried out using Immobiline DryStrips with a linear $\mathrm{pH}$ gradient between 4 and $7(7 \mathrm{~cm})$ on an IPGphor system (GE Healthcare) and proteins were separated by SDS-PAGE at $160 \mathrm{~V}$ for $2.5 \mathrm{~h}$. Spots were visualized by silver staining. Protein patterns in the gels were recorded as digitized images using a GS-800 imaging densitometer (Bio-Rad). Spot detection, matching and the examination of differentially expressed proteins were performed by PDQuest v. 8.0.1 software (Bio-Rad). Two biological and three technical replicates were made per condition and formed one replicate group with average normalized spot intensities. Comparison was carried out between cell-wall fractions from stationary-phase cells grown in MRS medium and in MRS in the presence of $1.2 \mathrm{~g}$ bile salts $1^{-1}$ (oxgall; Difco). A threshold of at least two times enhanced or decreased expression level was chosen to consider the differentially expressed proteins. The Plg-binding proteins were identified by comparison of the two-dimensional gels with the reference map reported by Candela et al. (2007).

MALDI-TOF-MS analysis of Plg binding to $B$. animalis subsp. lactis BI07. Plg binding to B. animalis subsp. lactis BI07 cells was evaluated by using a MALDI-TOF-MS-based approach, as reported by Candela et al. (2008a). Stationary-phase B. animalis subsp. lactis BI07 cells $\left(1 \times 10^{9}\right.$ c.f.u. $)$ grown in MRS with and without $1.2 \mathrm{~g}$ bile salts $1^{-1}$ (oxgall; Difco) were incubated with 0, 10, 20, 50, 100 and $200 \mu \mathrm{g}$ human Plg (Sigma-Aldrich). Subsequently, bifidobacteria were washed three times with PBS to remove unbound Plg. After washing, bacterial cells were resuspended in $20 \mu \mathrm{l} 2 \% \mathrm{NH}_{4} \mathrm{Cl}$ for MALDI-TOF analysis. In order to detect Plg recruited on the bacterial cell surface, $1.0 \mu \mathrm{l}$ of each bacterial suspension was spotted, airdried, and then overlaid with $1.0 \mu \mathrm{l}$ matrix solution $(12.5 \mathrm{mg}$ ferulic acid $\mathrm{ml}^{-1}$ in a mixture of formic acid/acetonitrile/water $\left.17: 33: 50\right)$. The resulting droplet was left to crystallize by airdrying and analysed in a Voyager DE Pro MALDI-TOF mass spectrometer (Applied Biosystems) operating in linear positive ion mode in the range of 20000-100000 Da and equipped with a pulsed $\mathrm{N}_{2}$ laser at $337 \mathrm{~nm}$. Default operating conditions were as follows: accelerating voltage $25000 \mathrm{~V}$, grid voltage $22500 \mathrm{~V}$, extraction delay time $750 \mathrm{~ns}$. All acquisitions were generated automatically on the instrument software (Voyager, Biospectrometry Workstation) using a random search pattern mode and based on overhanging 1600 shots from 20 nonoverlapping positions ( 80 shots per position). Data were externally calibrated with a standard BSAe (BSA) solution $(67 \mathrm{kDa})$. As analytical standard, $200 \mathrm{ng}$ human Plg were spotted. For each Plg concentration three biological and three technical replicates were carried out.

In order to evaluate the impact of DnaK and enolase on $B$. animalis subsp. lactis BI07 Plg-binding activity, Plg-binding assay of whole $B$. animalis subsp. lactis BI07 cells was performed after pre-treatment of cells with cross-reactive anti-meningococcal DnaK antiserum (Knaust et al., 2007) or anti-pneumococcal enolase antiserum (anti-Eno) (Bergmann et al., 2003), respectively. To this aim, $1 \times 10^{9}$ c.f.u. stationary-phase B. animalis subsp. lactis BI07 cells were washed in PBS and incubated with anti-meningococcal DnaK antiserum diluted $1 / 250$ or anti-Eno diluted $1 / 100$ in PBS- $1 \%$ BSA, for $1 \mathrm{~h}$ at $25{ }^{\circ} \mathrm{C}$ under constant agitation. After two washings with PBS-1 \% BSA, cells were resuspended in PBS and incubated with $100 \mu \mathrm{g}$ human Plg (Sigma-Aldrich) for $30 \mathrm{~min}$ at $37^{\circ} \mathrm{C}$ with constant agitation. Subsequently, bifidobacteria were washed three times with PBS to remove unbound Plg. After washing, bacterial cells were resuspended in $20 \mu \mathrm{l} \mathrm{NH}_{4} \mathrm{Cl} 2 \%$ for MALDI-TOF analysis of Plg binding, as previously described. For each experimental condition three biological and three technical replicates were carried out.
Plasmin activity assay. This was carried out as reported by Candela et al. (2008b). Briefly, stationary-phase B. animalis subsp. lactis BI07 cells grown in MRS with and without $1.2 \mathrm{~g}$ bile salts $1^{-1}$ (oxgall; Difco) were washed with PBS, adjusted to a concentration of $1 \times 10^{9}$ c.f.u. $\mathrm{ml}^{-1}$ and incubated for $30 \mathrm{~min}$ at $37^{\circ} \mathrm{C}$ with $20 \mu \mathrm{g} \mathrm{Plg}$ $\mathrm{ml}^{-1}$ (Sigma-Aldrich) in PBS. After two PBS washing steps, cells were resuspended in $50 \mathrm{mM}$ Tris/ $\mathrm{HCl}, \mathrm{pH} 7.5$ (TBS). A volume of $100 \mu \mathrm{l}$ of the bacterial cell suspension was added per well of a 96-well microtitre plate. Plg was activated with $0.06 \mathrm{KIU}$ urokinase PA (uPA) (Calbiochem), and $30 \mu \mathrm{l}$ of plasmin-specific chromogenic substrate solution, containing $0.54 \mathrm{mM}$ D-valyl-leucyl-lysine- $p$-nitroanilide dihydrochloride (S-2251) (Sigma-Aldrich), was added. Absorbance at $405 \mathrm{~nm}$ was measured by using a Multiskan Ascent V1.24 (Thermo Electron Corporation) immediately after the addition of S-2251 and at intervals of $7 \mathrm{~min}$ during $1 \mathrm{~h}$ incubation at $37^{\circ} \mathrm{C}$. As negative control the kinetic experiment was carried out with $B$. animalis subsp. lactis BI07 cells not incubated with Plg. Further controls were performed with B. animalis subsp. lactis BI07 cells incubated with $\mathrm{Plg}$ in the presence of $0.1 \mathrm{M}$ EACA (Sigma-Aldrich) and in the absence of uPA. Controls for spontaneous hydrolysis of S-2251 were carried out with the chromogenic substrate alone and in the presence of PA. Experiments were repeated twice and 10 technical replicates were carried out for each condition

\section{RESULTS}

\section{Localization of DnaK protein on the cell surface of B. animalis subsp. lactis $\mathrm{B} 107$}

The presence of the DnaK protein in the cytoplasmic and cell-wall fractions of $B$. animalis subsp. lactis BI07 was demonstrated by immunoblot analysis using a crossreactive anti-meningococcal DnaK antiserum (anti-DnaK) (Knaust et al., 2007) (Fig. 1). Immunoelectron microscopy was conducted to confirm the presence of DnaK on the surface of the bacteria. B. animalis subsp. lactis BI07 cells were incubated under pre-embedding labelling conditions with the anti-DnaK antiserum followed by the secondary antibody labelled with $10 \mathrm{~nm}$ gold particles. Analysis of ultrathin sections at a magnification of $\times 22000$ showed the presence of DnaK in the bacterial cell-wall region (black dots, arrowed in Fig. 2). No non-specific binding of the secondary antibody was detected (data not shown).

\section{Analysis of Plg binding to B. animalis subsp. lactis BI07 DnaK}

A genomic library of $B$. animalis subsp. lactis BI07 was constructed and screened for the $d n a K$ gene by using a digoxigenin-labelled DNA probe targeting the $B$. longum NCC 2705 dnaK gene. The nucleotide sequence of $B$. animalis subsp. lactis BI07 dnaK (GenBank accession no. AB514431) showed $100 \%$ identity with the dnaK gene of $B$. animalis subsp. lactis DSM 10140 (GenBank accession no. CP001606) and $87 \%$ with that of B. longum NCC 2705 (GenBank accession no. AE014295). In order to study the specific Plg-binding activity of $B$. animalis subsp. lactis BI07 DnaK, a His 6 -tagged DnaK protein was expressed and, after purification, a solid-phase Plg-binding assay was carried out (Sanderson-Smith et al., 2007). The binding 


\section{(a)}

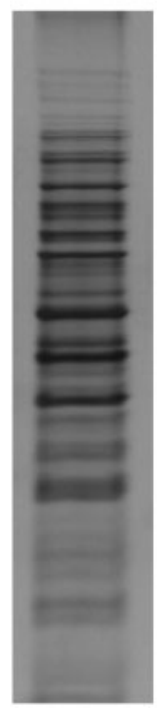

(b)

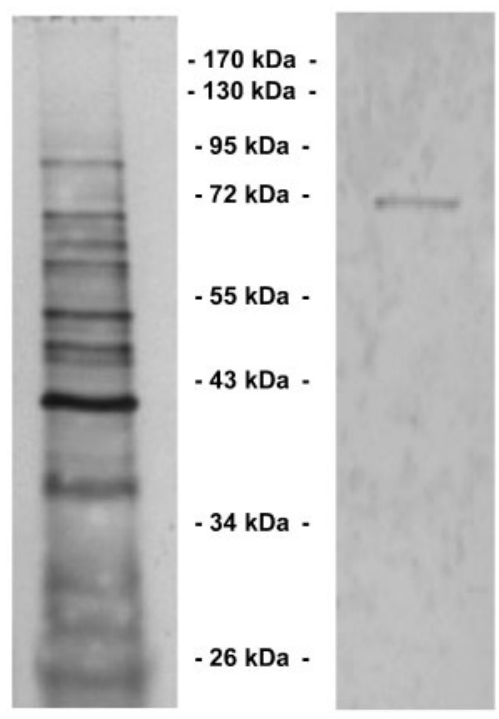

Fig. 1. Immunoblot analysis (right panels) using anti-DnaK antiserum of cytoplasmic (a) and cell wall (b) protein fractions of $B$. animalis subsp. lactis BI07 resolved by SDS-PAGE. (right panels).

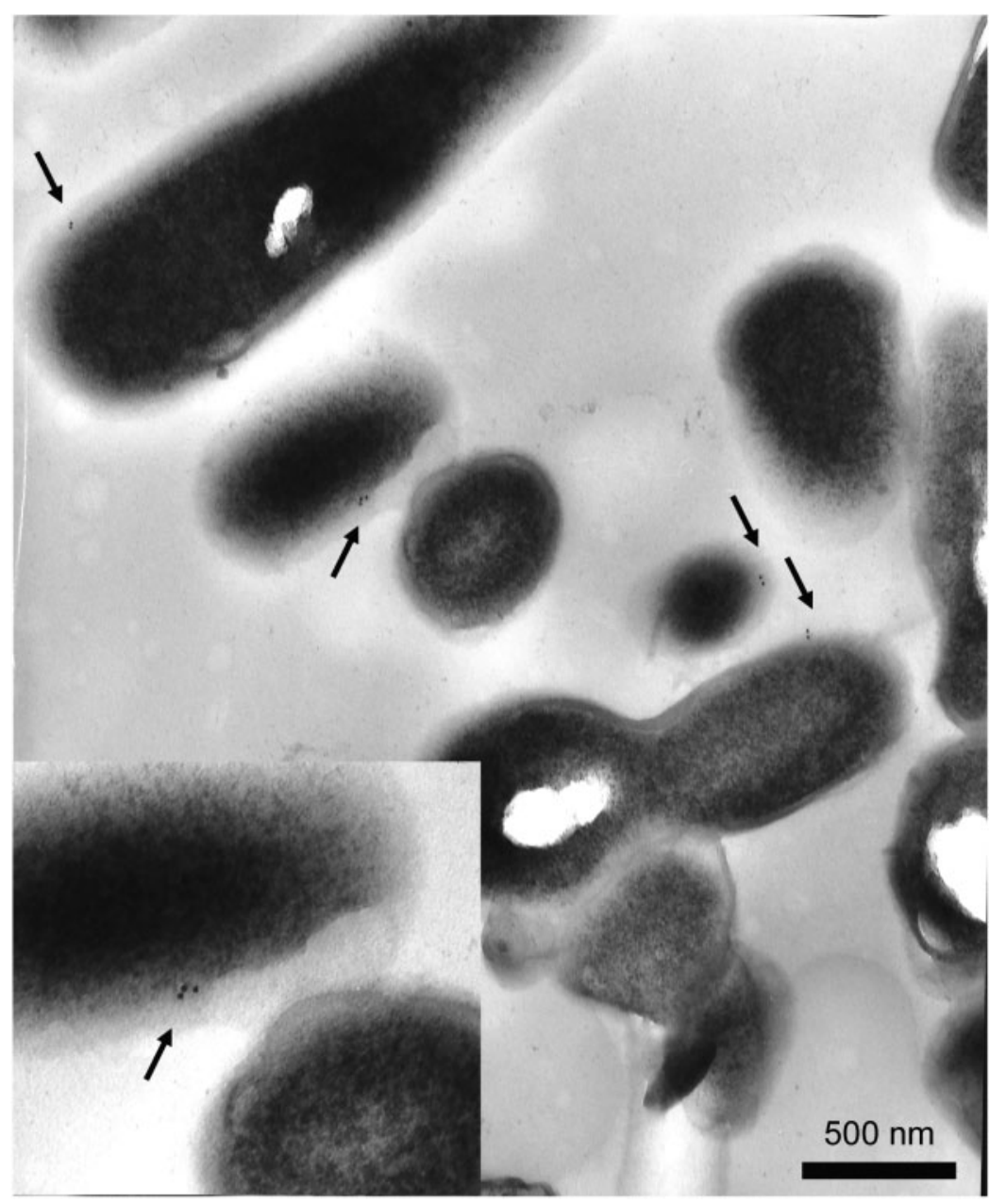

Fig. 2. Immunoelectron microscopic localization of DnaK on the cell surface of $B$. animalis subsp. lactis BI07. DnaK was detected on the bacterial surface by antiDnaK antiserum and secondary antibody coupled to $10 \mathrm{~nm}$ gold particles in preembedding experiments. An ultrathin section shows DnaK (black dots, arrowed) localized directly in the cell-wall region. 
assay demonstrated a dose-dependent interaction between biotinylated $\mathrm{Plg}$ and immobilized recombinant $B$. animalis subsp. lactis BI07 DnaK (Fig. 3). In the presence of 150 $\mathrm{nM}$ Plg a saturation binding was achieved. Non-specific binding was determined in the presence of 50 -fold molar excess of unlabelled $\mathrm{Plg}$, and specific binding was calculated by subtracting non-specific binding from the total binding obtained at each concentration of biotinylated Plg. As negative control BSA-coated wells were utilized. The bestfit non-linear regression analysis allowed calculation of an equilibrium dissociation constant $\left(K_{\mathrm{D}}\right)$ for the interaction with Plg of about $11 \mathrm{nM}$. The pivotal role of DnaK lysine residues in $\mathrm{Plg}$ binding was demonstrated with $0.5 \mathrm{M}$ EACA as competitor, which completely inhibited $\mathrm{Plg}$ binding (Fig. 3).

\section{Role of surface DnaK and enolase in Plg binding to $B$. animalis subsp. lactis $\mathrm{Bl} 107$}

In order to provide experimental evidence of the role of surface DnaK and enolase as Plg receptors, we evaluated the impact of pre-treatment with anti-DnaK or antienolase antibodies on B. animalis subsp. lactis BI07 Plgbinding activity. Bacterial cells were pre-treated with antiDnaK or anti-Eno (Candela et al., 2009) antisera and their Plg-binding activity was compared with that of untreated bacteria using MALDI-TOF analysis. As a control, $B$. animalis subsp. lactis BI07 cells not treated with Plg were utilized. The results showed that treatment with the antiEno antiserum decreased the Plg-binding activity by about $30 \%$, and treatment with the meningococcal anti-DnaK

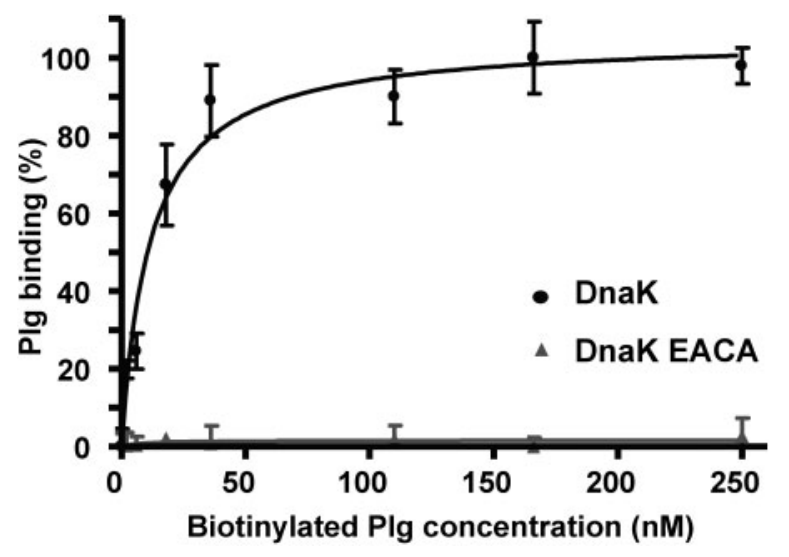

Fig. 3. Saturation binding analysis of biotinylated Plg to immobilized recombinant $B$. animalis subsp. lactis BI07 His-tagged DnaK. The black dots represent the specific binding. Specific Plg binding was calculated at each concentration of biotinylated Plg by subtracting non-specific binding obtained in the presence of 50 fold molar excess of unlabelled $\mathrm{Plg}$ from total binding. A one-site hyperbolic binding function was fitted to the data and the $K_{\mathrm{D}}$ determined. The experiment was repeated in the presence of the lysine analogue, EACA (0.5 M, grey triangles). Values represent the mean \pm SD of 9 measurements. antiserum decreased it by about $80 \%$ (Fig. 4). In contrast, no reduction in $\mathrm{Plg}$ binding was measured when using anti-rabbit IgG as blocking antibodies. These results suggest that both enolase and especially DnaK are of importance for the sequestration of plasminogen by $B$. animalis subsp. lactis B107.

\section{Impact of bile salts exposure on the $B$. animalis subsp. lactis BI07-plasmin(ogen) interaction}

First, the capability of $B$. animalis subsp. lactis BI07 to grow in the presence of bile salts was tested. To this end, $B$. animalis subsp. lactis BI07 was grown in batch cultures in the absence and in the presence of bile salts at concentrations of 1.2 and $3 \mathrm{~g} \mathrm{l}^{-1}$ (Sanchez et al., 2005). As reported for B. longum NCIMB 8809 , growth rates were very similar during the exponential phase in each of the three different conditions (Fig. 5). In contrast, at the end of the stationary-phase the c.f.u. $\mathrm{ml}^{-1}$ were significantly lower in the presence of bile salts $\left(1.2 \times 10^{9}, 5 \times 10^{8}\right.$ and $1 \times 10^{8}$ c.f.u. $\mathrm{ml}^{-1}$ for bile salts concentrations of $0,1.2$ and 3 $\mathrm{g}^{-1}$, respectively). In order to assess whether the physiological changes that are a consequence of the longterm exposure of B. animalis subsp. lactis BI07 to bile salts, can affect the expression of surface Plg receptors, a comparative proteomic approach was performed. The cell-wall proteomes of B. animalis subsp. lactis BI07 grown in media supplemented or not supplemented with bile salts at the concentration of $1.2 \mathrm{~g} \mathrm{l}^{-1}$ were compared by twodimensional gel electrophoresis (Fig. 6). Plg-binding proteins are numbered as reported by Candela et al. (2007). The expression profiles showed that exposure to bile salts induced a 2.8-, 2.3- and 10-fold upregulation of three Plg-binding proteins: DnaK, enolase and phosphoglycerate mutase, respectively. Surprisingly, the putative Plg-binding protein BSH was slightly downregulated (by about 1.7-fold) after bile exposure. A downregulation was also observed for the as yet unidentified Plg-binding proteins no. 2 and no. 8.

To investigate whether bile salts adaptation affects the Plgbinding capacity of $B$. animalis subsp. lactis $\mathrm{BI} 07$, we compared the Plg-binding activity of stationary-phase cells grown in the presence or in the absence of $1.2 \mathrm{~g}$ bile salts $\mathrm{I}^{-1}$. B. animalis subsp. lactis BI07 cells were incubated with different concentrations of Plg and the bacterial-bound $\mathrm{Plg}$ was measured by MALDI-TOF (Fig. 7). Although B. animalis subsp. lactis BI07 showed a dose-dependent Plgbinding activity independently of bile salts supplementation, bacteria grown in the presence of bile salts were about 10 times more efficient in Plg binding. In a plasmin activity assay we compared the capability of $B$. animalis subsp. lactis BI07 cells grown with and without $1.2 \mathrm{~g}$ bile salts $\mathrm{l}^{-1}$ to acquire a Plg-dependent surface-associated plasmin activity after the addition of host PA. To this end, $B$. animalis subsp. lactis BI07 stationary-phase cells were incubated with $20 \mu \mathrm{g} \mathrm{Plg} \mathrm{ml}{ }^{-1}$ and the cell-captured Plg was then activated to plasmin by the addition of uPA. The 


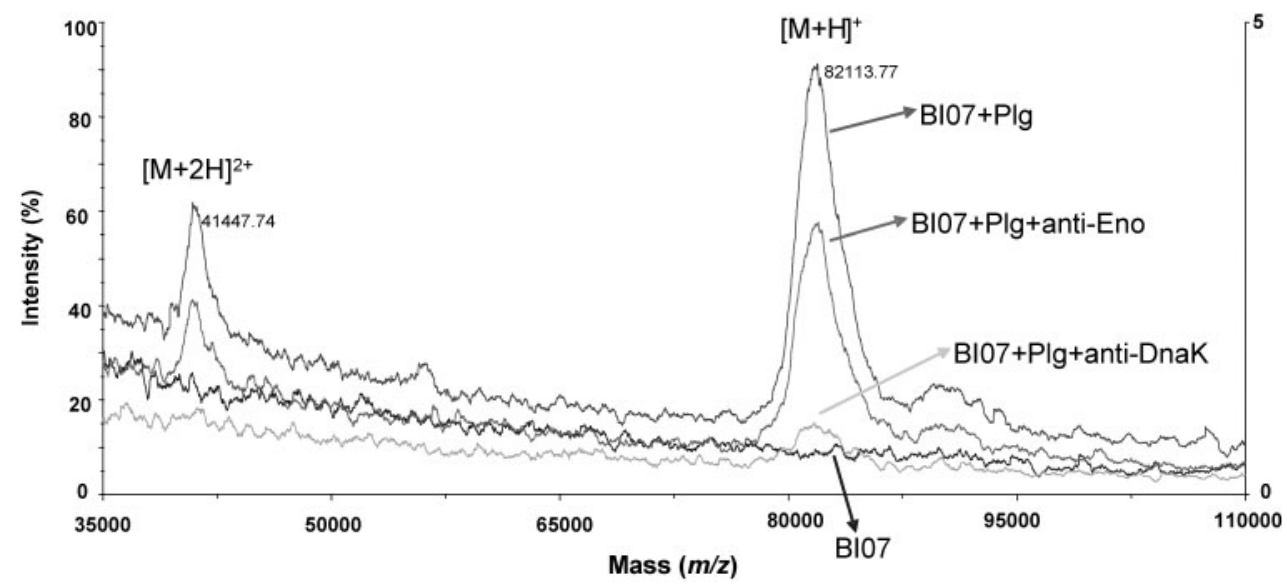

Fig. 4. MALDI-TOF analysis of Plg binding to $B$. animalis subsp. lactis BIO7 cells untreated (BIO7+Plg), and pre-treated with anti-DnaK (BI07+Plg + anti-DnaK) or anti-Eno (BI07+Plg + anti-Eno). As negative control, B. animalis subsp. lactis BI07 cells not incubated with Plg (BI07) are shown. Plg $[\mathrm{M}+\mathrm{H}]^{+}$signal at $81 \mathrm{kDa},[\mathrm{M}+2 \mathrm{H}]^{2+}$ at $40 \mathrm{kDa}$.

acquired bacterial surface-associated plasmin activity was evaluated in kinetic experiments by measuring the hydrolysis of the plasmin-specific chromogenic substrate S-2251 at intervals of 7 min over a period of $1 \mathrm{~h}$ (Fig. 8). The B. animalis subsp. lactis BI07 cells grown in the presence of bile salts showed a significant increase in the rate of S-2251 hydrolysis, demonstrating a higher rate of surface-associated Plg-derived plasmin activity compared with cells grown in standard conditions. As expected, the lysine analogue EACA was effective in inhibition of the plasmin formation of the B. animalis subsp. lactis BI07 cell

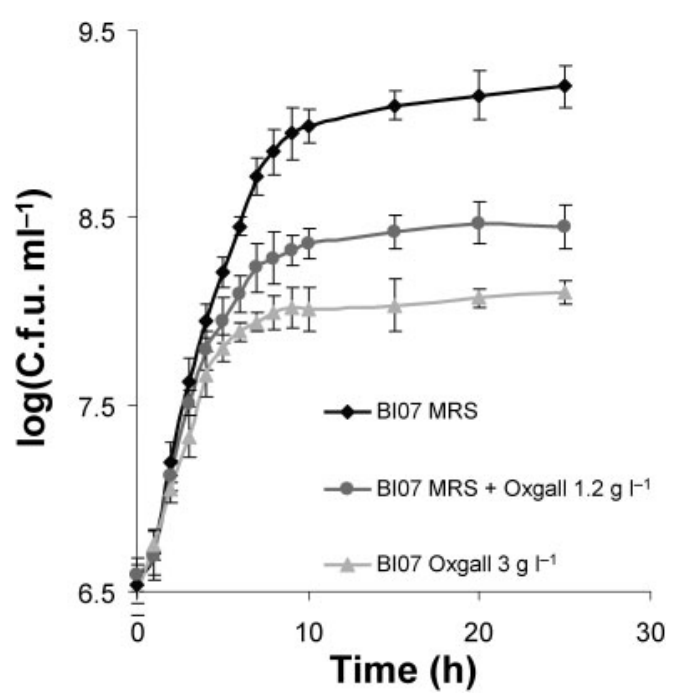

Fig. 5. Growth curves of $B$. animalis subsp. lactis $B I 07$ in MRS with 0 (black), 1.2 (dark grey) and $3 \mathrm{~g} \mathrm{I}^{-1}$ bile salts (light grey). For all the experimental conditions growth curves were performed in three biological replicates and values represent the mean \pm SD. surface, proving the essential role of the Plg lysine-binding sites for its recruitment to the bacterial cell surface (Fig. 8).

\section{DISCUSSION}

In this work we demonstrate the importance of $B$. animalis subsp. lactis BI07 DnaK as a surface receptor for human Plg. DnaK is a highly conserved primary cytoplasmic protein of $67 \mathrm{kDa}$ belonging to the heat-shock protein 70 family (HSP70) (Bukau \& Horwich, 1998). HSP70s are chaperones with an important role in protein folding and transport. By immunoelectron microscopy we documented the presence of DnaK on the cell surface of $B$. animalis subsp. lactis BI07. Similarly to the surface-located $B$. animalis subsp. lactis BI07 enolase (Candela et al., 2009), DnaK lacks predicted protein-sorting elements and anchorage on the bacterial cell wall. Mechanisms of secretion and surface localization of these cytoplasmic enzymes are still under debate. However, for Streptococcus pneumoniae, scavenging of cytoplasmic proteins released through allolysis was proposed as a possible mechanism for surface localization (Claverys \& Havarstein, 2007). Strengthening our findings, DnaK had been previously shown to be present on the cell surface of several pathogens such as Neisseria meningitidis, Listeria monocytogenes and Mycobacterium tuberculosis (Schaumburg et al., 2004; Knaust et al., 2007; Xolalpa et al., 2007), as well as the probiotic member of the human intestinal microbiota Lactobacillus salivarius (Kelly et al., 2005). Although for $L b$. salivarius no information had been provided concerning the DnaK function on the cell surface, in the case of $N$. meningitidis, L. monocytogenes and M. tuberculosis its role as receptor for human Plg was proved. The His-tagged $B$. animalis subsp. lactis BI07 DnaK protein was purified and its affinity for human Plg was evaluated in a saturation binding analysis. According to our data, the B. animalis 
(a)

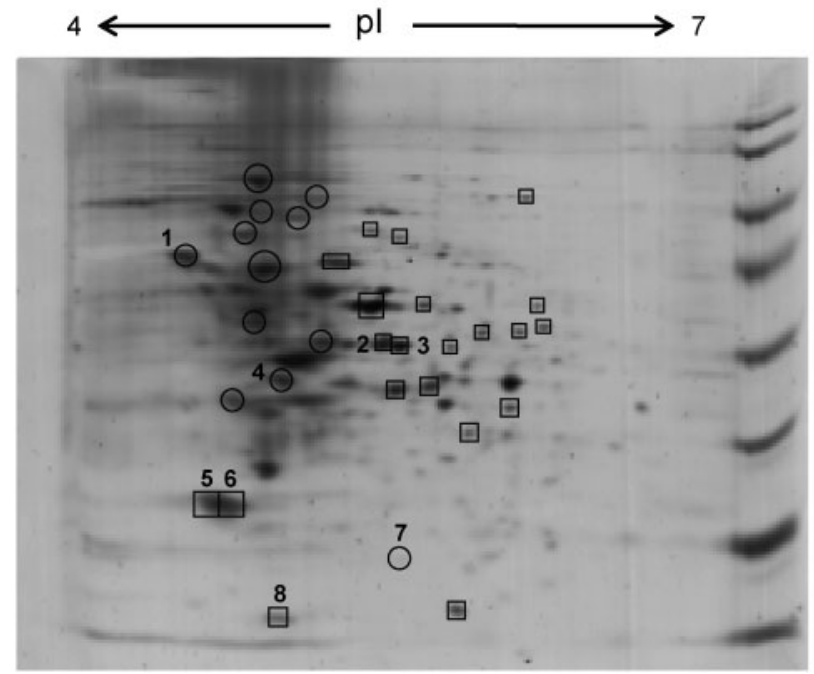

(b)

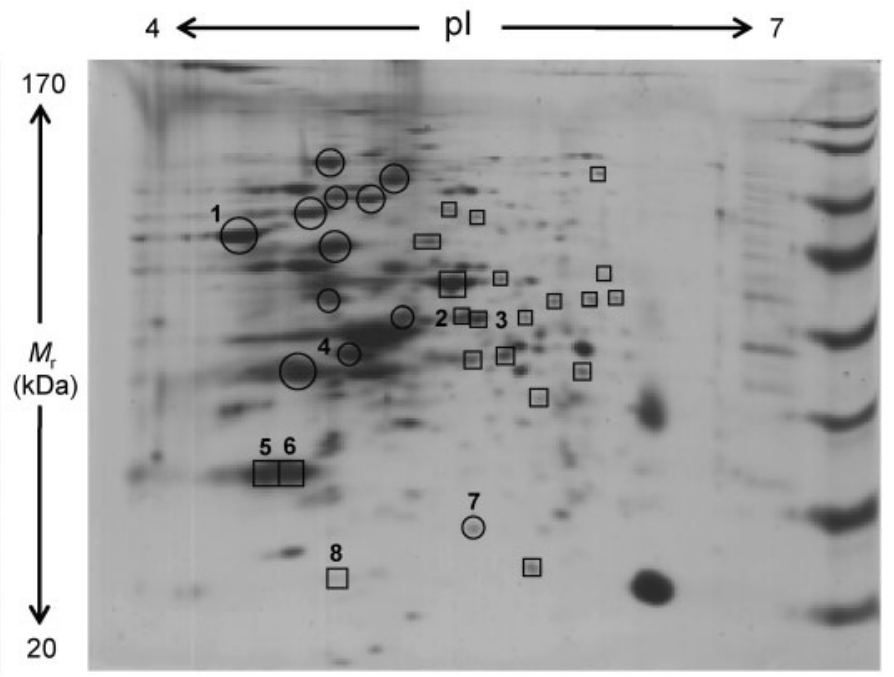

Fig. 6. Cell-wall proteome of stationary-phase B. animalis subsp. lactis BI07 cells cultured with (b) and without (a) bile salts at $1.2 \mathrm{~g} \mathrm{l}^{-1}$. Cell-wall proteins up- and downregulated as a consequence of bile salts adaptation are marked with circles and squares, respectively. Plg-binding proteins are numbered: DnaK (1), unidentified (2), glutamine synthetase (3), enolase (4), $\mathrm{BSH}(5,6)$, phosphoglycerate mutase (7), unidentified (8).

subsp. lactis BI07 DnaK shows a high affinity for human Plg with a $K_{\mathrm{D}}$ of about $11 \mathrm{nM}$, a value significantly higher than that reported for B. animalis subsp. lactis BI07 enolase $\left(K_{\mathrm{D}}=42.8 \mathrm{nM}\right)$ (Candela et al., 2009). The complete inhibition of Plg binding in the presence of $0.5 \mathrm{M}$ EACA indicates that DnaK lysine residue(s) are crucial for the $\mathrm{Plg}$ interaction. The pretreatment of B. animalis subsp. lactis BI07 cells with cross-reactive DnaK-specific antiserum had a detrimental impact on the bacterial Plg-binding capacity,

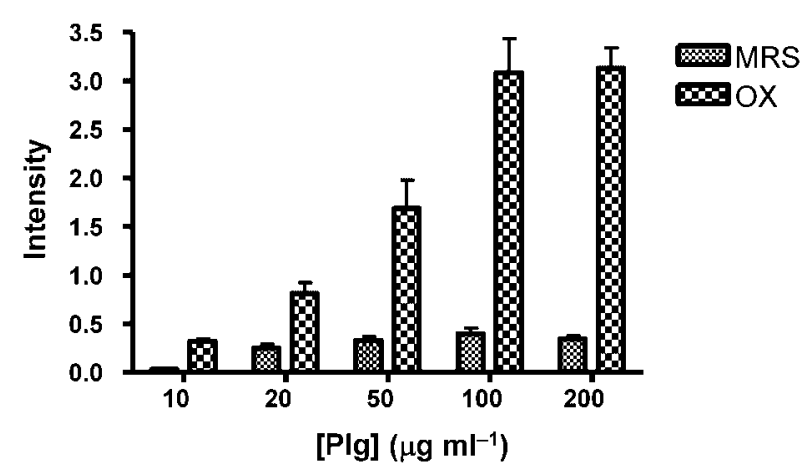

Fig. 7. MALDI-TOF analysis of the Plg-binding activity of stationary-phase cells of $B$. animalis subsp. lactis BI07 grown in the presence (OX) or in the absence (MRS) of $1.2 \mathrm{~g}$ bile salts $\mathrm{I}^{-1}$. The bars represent the response, expressed as Plg amplitude/ reference signal amplitude (RS amplitude), with the standard deviation, of $B$. animalis subsp. lactis $\mathrm{BIO} 7$ cells incubated with different concentrations of $\mathrm{Plg}\left(10,20,50,100,200 \mu \mathrm{g} \mathrm{ml}^{-1}\right)$. Reference signal: $23000 \mathrm{Da}$. demonstrating the importance of DnaK as a surface Plg receptor. Pre-treatment with anti-Eno also reduced $\mathrm{Plg}$ binding to $B$. animalis subsp. lactis BI07, but by only $30 \%$, compared to the $80 \%$ reduction seen with anti-DnaK antiserum. While it would be tempting to speculate that this difference is due to the higher Plg affinity of DnaK compared to enolase, we can not exclude that it is the consequence of a different efficacy in blocking the respective antigens between the two antisera. Moreover, the contribution of a single $\mathrm{Plg}$ receptor to Plg recruitment on the bacterial cell surface can be influenced by other variables alongside the mere affinity for $\mathrm{Plg}$, such as its availability on the cell surface and the number of binding epitopes (Knaust et al., 2007).

Adaptation of B. animalis subsp. lactis BI07 to bile salts induces a complex physiological response which influences the cell envelope proteome. Similarly to B. longum (Ruiz et al., 2009), the long-term exposure of B. animalis subsp. lactis $\mathrm{BI} 07$ to bile salts results in the upregulation of the important surface $\mathrm{Plg}$ receptors DnaK and enolase. Supporting these findings, a recent study of the $B$. animalis subsp. lactis protein response to bile salts reported the upregulation of DnaK (Sanchez et al., 2007). In order to investigate if the physiological changes as a consequence of bile salts response could have an impact on the $B$. animalis subsp. lactis-Plg interaction, we compared the Plg-binding activity and surface-associated Plg-dependent plasmin activity of B. animalis subsp. lactis BI07 cells grown with and without $1.2 \mathrm{~g}$ bile salts $\mathrm{l}^{-1}$. According to our data, the B. animalis subsp. lactis BI07 cells grown in the presence of bile salts were about 10 times more efficient in Plg binding 


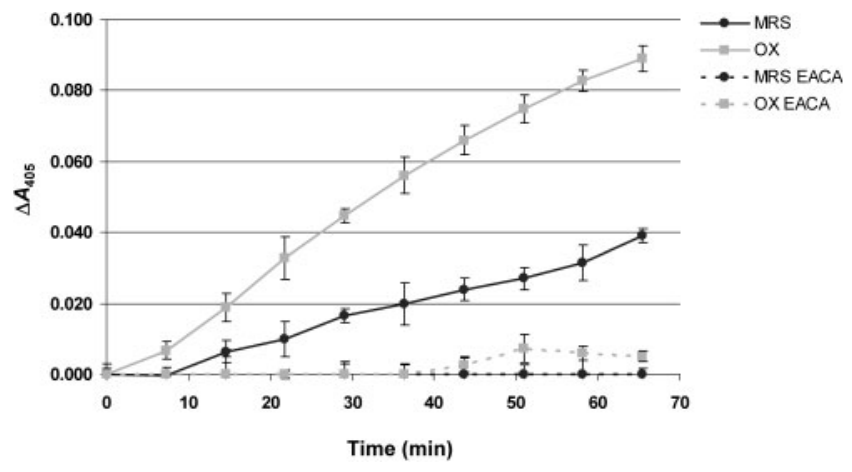

Fig. 8. Plasmin activity of $B$. animalis subsp. lactis $\mathrm{BI} 07$ cells grown in the presence (OX) or in the absence (MRS) of $1.2 \mathrm{~g}$ bile salts $\mathrm{I}^{-1}$. Plg-pretreated bacterial cells were incubated with uPA and then with the plasmin-specific chromogenic substrate S2251. The kinetics of plasmin formation was evaluated by measuring the increase in $A_{405}$ at intervals of $7 \mathrm{~min}$ during $1 \mathrm{~h}$ incubation. Bacterial cells untreated with Plg were used as negative control. Experiments were repeated by incubating the bacteria with Plg in the presence of $0.1 \mathrm{M} \mathrm{EACA}$. Values represent the mean \pm SEM of 20 measurements.

compared to cells cultured in standard medium and, after the addition of host uPA, these bacteria exhibited a significantly higher rate of acquired Plg-dependent surfaceassociated plasmin activity. Taken together, these data prove that bile salts enhance the ability of $B$. animalis subsp. lactis BI07 to interact with the host Plg system, and show the potential impact of the GIT bile environment on the $B$. animalis subsp. lactis-host interaction process. Even if the upregulation of surface Plg receptors such as enolase and DnaK can partially explain this increase in Plg-binding activity, other mechanisms may be involved. For instance, affecting the accessibility of Plg receptors, changes in the $B$. animalis subsp. lactis cell-surface structure may occur in response to bile adaptation (Ruiz et al., 2007) and may influence the bacterial affinity for human Plg (Stie et al., 2009).

Besides the demonstration of the importance of DnaK as a surface Plg receptor, in this work we report for the first time that $B$. animalis subsp. lactis significantly increases its affinity for human $\mathrm{Plg}$ in response to physiological concentrations of bile salts. Since the ability of Bifidobacterium to interact with the plasmin(ogen) system of the human host may have a role in facilitating the colonization of the human GIT (Candela et al., 2008b), our results provide evidence that the adaptation of $B$. animalis subsp. lactis to the gut bile environment may favour its capacity to colonize the human GIT. Further studies will be necessary to determine the mechanisms underlying the enhancement of B. animalis subsp. lactis BI07 Plg affinity in the presence of bile salts, as well as how bile influences the biology of the interaction process between $B$. animalis subsp. lactis BI07 and the host enterocytes. However, our findings provide some insights into the adaptation of $B$. animalis subsp. lactis to the human gastrointestinal environment. Moreover, the importance of DnaK as a $B$. animalis subsp. lactis Plg receptor justifies further studies to attempt to characterize the mechanisms of Plg binding in more detail.

\section{ACKNOWLEDGEMENTS}

We thank Matthias Frosch and Andreas Knaust (Institute of Hygiene and Microbiology, University of Wuerzburg, Germany) for providing the polyclonal anti-meningococcal DnaK antiserum.

\section{REFERENCES}

Barrangou, R., Briczinski, E. P., Traeger, L. L., Loquasto, J. R., Richards, M., Horvath, P., Coute-Monvoisin, A. C., Leyer, G., Rendulic, S. \& other authors (2009). Comparison of the complete genome sequences of Bifidobacterium animalis subsp. lactis DSM 10140 and Bl-04. J Bacteriol 191, 4144-4151.

Bergmann, S. \& Hammerschmidt, S. (2007). Fibrinolysis and host response in bacterial infections. Thromb Haemost 98, 512-520.

Bergmann, S., Wild, D., Diekmann, O., Frank, R., Bracht, D., Chhatwal, G. S. \& Hammerschmidt, S. (2003). Identification of a novel plasmin(ogen)-binding motif in surface displayed alpha-enolase of Streptococcus pneumoniae. Mol Microbiol 49, 411-423.

Bergmann, S., Rohde, M., Preissner, K. T. \& Hammerschmidt, S. (2005). The nine residue plasminogen-binding motif of the pneumococcal enolase is the major cofactor of plasmin-mediated degradation of extracellular matrix, dissolution of fibrin and transmigration. Thromb Haemost 94, 304-311.

Bernstein, H., Payne, C. M., Bernstein, C., Schneider, J., Beard, S. E. \& Crowley, C. L. (1999). Activation of the promoters of genes associated with DNA damage, oxidative stress, ER stress, and protein malfolding by the bile salt, deoxycholate. Toxicol Lett 108, 37-46.

Bukau, B. \& Horwich, A. L. (1998). The Hsp70 and Hsp60 chaperone machines. Cell 92, 351-366.

Candela, M., Bergmann, S., Vici, M., Vitali, B., Turroni, S., Eikmanns, B. J., Hammerschmidt, S. \& Brigidi, P. (2007). Binding of human plasminogen to Bifidobacterium. J Bacteriol 189, 5929-5936.

Candela, M., Fiori, J., Dipalo, S., Naldi, M., Gotti, R. \& Brigidi, P. (2008a). Rapid MALDI-TOF-MS analysis in the study of interaction between whole bacterial cells and human target molecules: binding of Bifidobacterium to human plasminogen. J Microbiol Methods 73, 276278.

Candela, M., Miccoli, G., Bergmann, S., Turroni, S., Vitali, B., Hammerschmidt, S. \& Brigidi, P. (2008b). Plasminogen-dependent proteolytic activity in Bifidobacterium lactis. Microbiology 154, 24572462.

Candela, M., Biagi, E., Centanni, M., Turroni, S., Vici, M., Musini, F., Vitali, B., Bergmann, S., Hammerschmidt, S. \& Brigidi, P. (2009). Bifidobacterial enolase, a cell surface receptor for human plasminogen involved in the interaction with the host. Microbiology 155, 32943303.

Claverys, J. P. \& Havarstein, L. S. (2007). Cannibalism and fratricide: mechanisms and raisons d'etre. Nat Rev Microbiol 5, 219-229.

Coleman, R., Iqbal, S., Godfrey, P. P. \& Billington, D. (1979). Membranes and bile formation. Composition of several mammalian biles and their membrane-damaging properties. Biochem J 178, 201208. 
Collen, D. \& Verstraete, M. (1975). Molecular biology of human plasminogen. II. Metabolism in physiological and some pathological conditions in man. Thromb Diath Haemorrh 34, 403-408.

Gill, S. R., Pop, M., Deboy, R. T., Eckburg, P. B., Turnbaugh, P. J., Samuel, B. S., Gordon, J. I., Relman, D. A., Fraser-Liggett, C. M. \& Nelson, K. E. (2006). Metagenomic analysis of the human distal gut microbiome. Science 312, 1355-1359.

Guarner, F. \& Malagelada, J. R. (2003). Gut flora in health and disease. Lancet 361, 512-519.

Hafkenscheid, J. C. \& Hectors, M. P. (1975). An enzymatic method for the determination of the glycine/taurine ratio of conjugated bile acids in bile. Clin Chim Acta 65, 67-74.

Hardie, K. \& Williams, P. (1998). Introduction: fractionation of bacterial cell envelopes. Methods Microbiol 27, 185-190.

Hurmalainen, V., Edelman, S., Antikainen, J., Baumann, M., Lähteenmäki, K. \& Korhonen, T. K. (2007). Extracellular proteins of Lactobacillus crispatus enhance activation of human plasminogen. Microbiology 153, 1112-1122.

Kelly, P., Maguire, P. B., Bennett, M., Fitzgerald, D. J., Edwards, R. J., Thiede, B., Treumann, A., Collins, J. K., O'Sullivan, J. C. \& other authors (2005). Correlation of probiotic Lactobacillus salivarius growth phase with its cell-wall associated proteome. FEMS Microbiol Lett 252, 153-159.

Klijn, A., Mercenier, A. \& Arigoni, F. (2005). Lessons from the genomes of bifidobacteria. FEMS Microbiol Rev 29, 491-509.

Knaust, A., Weber, M. V., Hammerschmidt, S., Bergmann, S., Frosch, M. \& Kurzai, O. (2007). Cytosolic proteins contribute to surface plasminogen recruitment of Neisseria meningitidis. J Bacteriol 189, 32463255.

Lähteenmäki, K., Kuusela, P. \& Korhonen, T. K. (2001). Bacterial plasminogen activators and receptors. FEMS Microbiol Rev 25, 531552.

Lähteenmäki, K., Edelman, S. \& Korhonen, T. K. (2005). Bacterial metastasis: the host plasminogen system in bacterial invasion. Trends Microbiol 13, 79-85.

Ley, R. E., Hamady, M., Lozupone, C., Turnbaugh, P. J., Ramey, R. R., Bircher, J. S., Schlegel, M. L., Tucker, T. A., Schrenzel, M. D. \& other authors (2008). Evolution of mammals and their gut microbes. Science 320, 1647-1651.

Meile, L., Ludwig, W., Rueger, U., Gut, C., Kaufmann, P., Dasen, G., Wenger, S. \& Teuber, M. (1997). Bifidobacterium lactis sp. nov., a moderately oxygen tolerant species isolated from fermented milk. Syst Appl Microbiol 20, 57-64.

Neish, A. S. (2009). Microbes in gastrointestinal health and disease. Gastroenterology 136, 65-80.

Parkkinen, J. \& Korhonen, T. K. (1989). Binding of plasminogen to Escherichia coli adhesion proteins. FEBS Lett 250, 437-440.

Ridlon, J. M., Kang, D. \& Hylemon, P. B. (2006). Bile salt biotransformations by human intestinal bacteria. J Lipid Res 47, 241-259.

Round, J. L. \& Mazmanian, S. K. (2009). The gut microbiota shapes intestinal immune responses during health and disease. Nat Rev Immunol 9, 313-323.

Ruiz, L., Sanchez, B., Ruas-Madiedo, P., de los Reyes-Gavilan, C. G. \& Margolles, A. (2007). Cell-envelope changes in Bifidobacterium animalis ssp. lactis as a response to bile. FEMS Microbiol Lett 274, 316-322.
Ruiz, L., Coutè, Y., Sanchez, B., de los Reyes-Gavilan, C. G., Sanchez, J. C. \& Margolles, A. (2009). The cell-envelop proteome of Bifidobacterium longum in an in vitro bile environment. Microbiology 155, 957-967.

Saksela, O. \& Rifkin, D. B. (1988). Cell-associated plasminogen activation: regulation and physiological functions. Annu Rev Cell Biol 4, 93-126.

Sanchez, B., Champomier-Verges, M. C., Anglade, P., Baraige, F., de los Reyes-Gavilan, C. G., Margolles, A. \& Zagorec, M. (2005). Proteomic analysis of global changes in protein expression during bile salt exposure of Bifidobacterium longum NCIMB 8809. J Bacteriol 187, 5799-5808.

Sanchez, B., Champomier-Verges, M. C., Stuer-Lauridsen, B., Ruas-Madiedo, P., Anglade, P., Baraige, F., de los Reyes-Gavilan, C. G., Johansen, E., Zagorec, M. \& Margolles, A. (2007). Adaptation and response of Bifidobacterium animalis subsp. lactis to bile: a proteomic and physiological approach. Appl Environ Microbiol 73, 6757-6767.

Sanderson-Smith, M. L., Walker, M. J. \& Ranson, M. (2006). The maintenance of high affinity plasminogen binding by group A streptococcal plasminogen-binding M-like protein is mediated by arginine and histidine residues within the al and a 2 repeat domains. J Biol Chem 281, 25965-25971.

Sanderson-Smith, M. L., Dowton, M., Ranson, M. \& Walker, M. J. (2007). The plasminogen binding group A streptococcal M proteinrelated protein Prp binds plasminogen via arginine and histidine residues. J Bacteriol 189, 1435-1440.

Schaumburg, J., Diekmann, O., Hagendorff, P., Bergmann, S., Rohde, M., Hammerschmidt, S., Jansch, L., Wehland, J. \& Karst, U. (2004). The cell wall subproteome of Listeria monocytogenes. Proteomics 4, 2991-3006.

Sha, J., Erova, T. E., Alyea, R. A., Wang, S., Olano, J. P., Pancholi, V. \& Chopra, A. K. (2009). Surface-expressed enolase contributes to the pathogenesis of clinical isolate SSU of Aeromonas hydrophila. J Bacteriol 191, 3095-3107.

Sijbrandi, R., Den Blaauwen, T., Tame, J. R. H., Oudega, B., Luirink, J. \& Otto, B. R. (2005). Characterization of an iron-regulated alphaenolase of Bacteroides fragilis. Microbes Infect 7, 9-18.

Stie, J., Bruni, G. \& Fox, D. (2009). Surface-associated plasminogen binding of Cryptococcus neoformans promotes extracellular matrix invasion. PLoS One 4, e5780.

Turroni, F., van Sinderen, D. \& Ventura, M. (2009). Bifidobacteria: from ecology to genomics. Front Biosci 14, 4673-4684.

Vassalli, J. D., Sappino, A. P. \& Belin, D. (1991). The plasminogen activator/plasmin system. J Clin Invest 88, 1067-1072.

Ventura, M., O'Flaherty, S., Claesson, M. J., Turroni, F., Klaenhammer, T. R., van Sinderen, D. \& O'Toole, P. W. (2009). Genome-scale analyses of health-promoting bacteria: probiogenomics. Nat Rev Microbiol 7, 61-71.

Xolalpa, W., Vallecillo, A. J., Lara, M., Mendoza-Hernadez, G., Comini, M., Spallek, R., Singh, M. \& Espitia, C. (2007). Identification of novel bacterial plasminogen-binding proteins in the human pathogen Mycobacterium tuberculosis. Proteomics 7, 33323341.

Zhang, L., Seiffert, D., Fowler, B. J., Jenkins, G. R., Thinnes, T. C., Loskutoff, D. J., Parmer, R. J. \& Miles, L. A. (2002). Plasminogen has a broad extrahepatic distribution. Thromb Haemost 87, 493-501.

Edited by: H. J. Flint 